\title{
PRINCIPALES FACTORES ASOCIADOS A LA INFRACCIÓN DE LA LEY PENAL COMETIDA POR LOS ADOLESCENTES EN EL DISTRITO JUDICIAL DE TACNA. 2013-2016
}

\author{
Main factors associated with the infringement of criminal law committed \\ by adolescents in the judicial district of Tacna. 2013-2016
}

PIERANN DEL ROSARIO FUENTES FUENTES 1

\section{Resumen}

Analiza el problema de la delincuencia juvenil, teniendo como objetivo determinar los principales factores que se encuentran asociados a la infracción de la ley penal cometida por los adolescentes en el distrito judicial de Tacna, 2013-2016. La hipótesis de estudio fue: En el distrito judicial de Tacna los principales factores que se encuentran asociados a la infracción de la ley penal cometida por los adolescentes, 20132016, son: sociales, económicos y jurídicos. Se utilizó el método de investigación básica con diseño no experimental de corte transversal. El estudio abarcó una población de 36 infracciones penales sentenciadas durante el período 20132016, así como 151 abogados que llevan casos de familia y 07 magistrados de familia de Tacna, para la recopilación de la información se aplicó tres instrumentos de medición (cuestionario, ficha de observación y la entrevista). Los resultados de la investigación permiten comprobar la hipótesis de estudio planteada.

Palabras claves: entorno familiar, adolescentes.

\section{Abstract}

Analyzes the problem of juvenile delinquency, aiming to determine the main factors associated with the crime Infraction of the criminal law committed by adolescents in the judicial district of Tacna, 2013-2016. The hypothesis of the study was: In the Judicial District of Tacna, the main factors that are associated with the infraction of the criminal law committed by adolescents, 20132016, are: social, economic and legal. We used the basic research method with non-experimental cross-sectional design. The study covered a population of 36 sentenced criminal offenses during the period 2013-2016, as well as 151 lawyers holding family cases and 07 Tacna family magistrates. Three measurement instruments were used for the information collection (questionnaire, Observation and interview). The results of the research allow to verify the proposed hypothesis of study.

Key words: Family environment, adolescents

\section{INTRODUCCIÓN}

El Estado tiene una atención prioritaria hacia los adolescentes debido a que la legislación peruana los protege de todas las formas posibles, en el entendido de que sus derechos son más fáciles de vulnerar, y de alguna manera si existiera algún problema serán sus derechos los que prevalecerán sobre los demás. Sin embargo, se ve a diario la violencia generada en la sociedad peruana, que muchas veces es ejercida por adolescentes, la delincuencia juvenil que podría tener como causa principal el nivel cultural que tienen los jóvenes. El problema de la familia

1 DOCTOR EN DERECHO PENAL Y POLITICA CRIMINAL 
constituye un factor que combinado con el factor socio económico puede contribuir a crear las condiciones favorables; pero no suficientes para el surgimiento de un comportamiento antisocial del adolescente.

La adolescencia implica un proceso de desarrollo que impulsa al joven a tomar sus propias decisiones sobre cómo pensar, sentir y actuar. Las decisiones más importantes de tomar en su vida son las referidas a temas familiares, luego amistades, estudios y sentimientos. Existe un alto incremento de infracciones a la ley penal por parte de los adolescentes; así, de acuerdo al estudio realizado por la Defensoría del Pueblo, en el año 2012 el $56.4 \%$ de adolescentes no ha completado la educación secundaria, el 23.2\% no ha culminado el nivel primario. En conclusión el nivel educativo de los adolescentes infractores de la ley penal muestra niveles más bajos frente al año 2007 (Defensoría del Pueblo, 2012)

Otra de las posibles causas del porqué hay jóvenes delincuentes, debemos centrarnos en el propio hecho antisocial. Por los que, los actos antisociales ocurren cuando los beneficios especiales superan a la desaprobación familiar, es decir, cuando un menor decide cometer una infracción éste ya ha valorado las posibilidades que tiene para conseguir sus objetivos, de los beneficios que conlleva la decisión que ha tomado.

Muchos adolescentes no se consideran responsables de sus experiencias, sino que todo lo atribuyen al destino, es decir, de todo aquello que les ocurre o lo que hacen, por ello tienden a auto exculparse y explicar su conducta como si ésta dependiera de personas o circunstancias ajenas a su control.

Reyna Castillo, 2011, se propone implantar la justicia restaurativa en adolescentes infractores y la utilización de la mediación como un método alternativo de solución de los conflictos de los adolescentes infractores reparando el daño causado y poder después ser reinsertado el adolescente en la sociedad. Propone evitar imponerse una pena privativa de la libertad. Logró obtener como resultado más importante, que en el Ecuador está muy poco difundida la justicia restaurativa, y, de serlo se lograría el descongestionamiento de la carga procesal. Miranda Cortes, 2011 pretende establecer en qué condiciones se encuentran los trabajadores sociales para desempeñarse en su trabajo en el sistema de ejecución de sanciones y medidas cautelares de menores infractores. Concluye que en el desarrollo de la práctica social de los trabajadores sociales utilizan los conocimientos aportados por su formación del pregrado en su quehacer profesional y realizan una multiplicidad de acciones de diversa índole que en el marco de las orientaciones técnicas los induce a construir su rol profesional apelando a su ética profesional y a sus concepciones sobre el abordaje técnico a la problemática de los jóvenes. Wiese, 2008, refiere que el problema investigado radica en la violencia de los jóvenes que provocan un impacto devastador en la sociedad. Concluye que la población integrada por jóvenes que cometieron homicidio ha estado expuesta a los mismos factores de riesgo que los que cometieron otro tipo de infracciones a la ley. Unos y otros resultan provenientes de la misma población, comparten similares antecedentes, vulnerabilidades sociales y psicológicas. Hernandez Alarcon C. A., 2005, pretende determinar las razones del divorcio entre la legislación internacional y el respeto de los derechos vinculados con la administración de justicia del adolescente sometido a la justicia penal juvenil en el Perú. Llegó a la conclusión de incluir en la legislación una regulación normativa que incluya la prevención como un elemento de política pública estatal.

El proceso penal que regula el juzgamiento de los adolescentes no sólo posibilita y legitima violaciones al debido proceso, sino que en su ejecución fracasa tanto desde la perspectiva del Estado como de la víctima y del mismo adolescente al favorecer la reincidencia. Dentro de un modelo acusatorio, no debe caerse en el excesivo reglamentarismo. Respecto a la educación , Juan Rivera Palomino refiere que la educación es un proceso social o histórico que se da en una sociedad determinada en un periodo o momento histórico, se procesa en contextos socio históricos concretos que son los que le otorgan un significado, características y funciones específicas. Walter Peñaloza: La educación encierra un triple proceso: hominización, socialización y culturización y Jorge Capella: La educación es un proceso de emancipación, mediante el cual los sujetos y los pueblos dejan de ser menos individuos para convertirse en agentes de su propio destino, gracias a su capacidad transformadora. El aprendizaje, es el rol más obvio y reconocido socialmente para los centros educativos donde se les enseña una serie de cosas que, a su vez, los niños supuestamente aprenden y 
ocasionalmente disfrutan (Trahtemberg, 1999,p.142). La educación es un mecanismo a través del cual se prepara la mano de obra que necesita el sistema o estructura económica (Vera y Bedregal,2010,p.78). La adquisición de ideas, conceptos, destrezas, hábitos, etc., es lo que constituye la educación. La educación escolar y familiar es considerada como uno de los medios más efectivos para prevenir lo antisocial (Hikal, 2005,p.102). El nivel educativo se considera parte de la formación de una persona y el comportamiento de una persona muestra su educación. (Gran influencia de los amigos en el entorno escolar, 2013). Es en la escuela o en el barrio en donde se facilitan ciertas relaciones sociales promiscuas que en la infracción juvenil tienen gran importancia y se denomina como malas compañías o a mistades (Hikal,2005,p.100). El niño que procede de una familia completa, estable, solvente, estimuladora tiene ventajas para aprovechar la educación escolarizada por razones socio culturales y de orden familiar que no las tiene el niño con carencias primarias, así vaya al mismo colegio que el otro (Trahtemberg,1999,p.145). Es asi que se sabe que las relaciones entre el aprendizaje y el pensamiento plantean gran número de problemas a la investigación criminológica (Hikal, 2005,p.114).

La familia es el núcleo social unido por vínculos de sangre o emergente del matrimonio, que regularmente se halla sometido a una dirección única y cuyos miembros hacen vida permanente bajo un techo común (Arias, 1952,p.178). La familia en sentido restringido, es el núcleo social conformado por todas las personas que habitan en una vivienda, bajo la autoridad de una persona (Hinostroza, 1999,p.80). La familia es un organismo jurídico y que representa una institución, posición sostenida por La Cruz y Antonio Cicu. Según esta doctrina la familia sería el conjunto de personas agrupadas de manera natural y necesaria y que por tal razón acompaña al Estado, aunque es anterior y superior a él. Esta posición es rechazada, por la comparación que se le hace a la familia con el Estado, lo cual produce la abstracción de la primera, donde los padres se deshumanizan, los cual desdice de la esencia misma del vínculo familiar (Hinostroza, 1999,p.105).La solidez de un Estado se funda en la estrecha unión que vincula al grupo familiar. De ser débiles los vínculos familiares lo será también el Estado; de ser fuerte aquellos, el Estado tendrá una base sólida y estable. (Diaz De Guijarro, 1943,p.156). Desde el punto de vista moral, fomentará la cultura cívica de los ciudadanos, lo que dará lugar a un clima de armonía y solidaridad de la Nación en su conjunto. (Diaz De Guijarro, 1943,p.156). Los padres cumplen un rol trascendente en los problemas de los adolescentes, en la mayoría de los casos es casi imposible reinsertar al joven delincuente en la sociedad si no se lo ha reacomodado en su grupo familiar. En algunas ocasiones son las propias familias las que empujan al niño o adolescente al conflicto (Kemelmajer, 2004,p.78). El entorno social durante la adolescencia es uno de los elementos que más influye en el desarrollo de la personalidad (Gran influencia de los amigos en el entorno escolar, 2013).

La investigación internacional afirma que la pobreza juega un rol en el desarrollo del comportamiento delictivo en la juventud. Una de las hipótesis indica que su influencia es indirecta, ya que actúa sobre la familia, la que, al sufrir de situaciones de marginalidad o pobreza, se ve expuesta a desarrollar problemas como depresión familiar, conflicto, hostilidad parental y desorganización de funciones familiares. El factor social que se asocia a la infracción de la ley penal de los adolescentes está íntimamente relacionado con el entorno familiar y con la participación comunitaria. En la mayoría de casos es imposible reinsertar al joven delincuente en la sociedad si previamente no se lo ha reacomodado en su grupo familiar, en algunas ocasiones son las propias familias las que empujan al delincuente hacia el conflicto. Las personas que rodean a los adolescentes muchas veces son personas que no tienen una formación profesional de valores y principios; ni competencia educativa específica; la delincuencia juvenil ha representado y representa preocupación fundamental en la criminología histórica y contemporánea, la cual queda justificada por la existencia real de situaciones objetivas debidamente comprobadas. Los positivistas como la criminología positiva contemporánea tienen una clara orientación determinista, pero no sólo respecto a los jóvenes, sino respecto a todo aquél que delinca. Los fenómenos adolescentes son hechos sociales, es decir hechos por la sociedad, producidos socialmente, producto de las relaciones sociales y por ello también sujeto de relaciones sociales, esto quiere decir, que la infancia y la adolescencia ha de ser comprendida y explicada desde la sociedad, de ahí con su relación con el nivel cultural. 


\section{OBJETIVOS}

- Establecer la asociación que existe entre el factor social y la infracción de la ley penal cometida por los adolescentes en el distrito judicial de Tacna, 2013-2016.

- Establecer la asociación que existe entre el factor económico y la infracción de la ley penal cometida por los adolescentes en el distrito judicial de Tacna, 2013-2016.

- Establecer la asociación que existe entre el factor jurídico y la infracción de la ley penal cometida por los adolescentes en el distrito judicial de Tacna, 2013-2016.

\section{METODOLOGÍA}

La investigación fue de tipo básica, no experimental de corte transversal, correlacional. El alcance de estudio abarcó los casos de infracción penal sentenciados en los tres juzgados de familia de la Corte Superior de Justicia del cercado de la ciudad de Tacna, período 2013-2016.
La población estuvo constituida por 36 casos de infracción a la ley penal.

La muestra de abogados estuvo constituida 151 abogados de familia; así como también por 3 jueces. Para realizar la investigación se utilizó el cuestionario, la ficha de observación y la cédula de entrevista.

\section{RESULTADOS}

El $64 \%$ de infracciones cometidas por los adolescentes corresponden a delitos contra el patrimonio, mientras que el $14 \%$ delitos contra la vida, el cuerpo y la salud, el $17 \%$ a delitos contra la seguridad pública y el $6 \%$ a delitos contra la fe pública (Tabla 01).

Tabla 01. Tipo de Infracción

\begin{tabular}{lrr}
\hline Tipo de infracción & $\mathrm{n}$ & $\%$ \\
\hline Delitos contra el patrimonio & 23 & $64 \%$ \\
\hline $\begin{array}{l}\text { Delitos contra la vida, el cuerpo } \\
\text { y la salud }\end{array}$ & 5 & $14 \%$ \\
\hline $\begin{array}{l}\text { Delitos contra la seguridad } \\
\text { pública }\end{array}$ & 6 & $17 \%$ \\
\hline Delitos contra la fe pública & 2 & $6 \%$ \\
\hline TOTAL & 36 & $100 \%$ \\
\hline
\end{tabular}

El $44 \%$ de infracciones cometidas por los adolescentes corresponden a robo, mientras que el $25 \%$ corresponde a hurto, el $11 \%$ a peligro común, el $8 \%$ de las infracciones corresponden a lesiones leves, el $6 \%$ a falsedad genérica, y el $6 \%$ de las infracciones corresponden a tráfico ilícito de drogas (Tabla 02).

\section{Tabla 02. Modalidad de la Infracción}

\begin{tabular}{lrr}
\hline Modalidad & $\mathrm{n}$ & $\%$ \\
\hline Robo & 16 & $44 \%$ \\
\hline Hurto & 9 & $25 \%$ \\
\hline Peligro común & 4 & $11 \%$ \\
\hline Lesiones leves & 3 & $8 \%$ \\
\hline Falsedad genérica & 2 & $6 \%$ \\
\hline Tráfico ilícito de drogas & 2 & $6 \%$ \\
\hline TOTAL & 36 & $100 \%$ \\
\hline
\end{tabular}

El $47 \%$ de infracciones penales fueron cometidas utilizando arma blanca, el 19\% utilizó sólo sus manos, el $8 \%$ otro tipo de objetos contundentes, mientras que el $25 \%$ no utilizó nada. El $39 \%$ de las infracciones penales se cometieron con lesiones, el $19 \%$ con maltrato 
psicológico hacia la víctima, el $17 \%$ con maltrato sin lesión, mientras que el $25 \%$ con otros daños producidos al Estado, a la salud y a la seguridad del adolescente. El $56 \%$ de infracciones penales fueron cometidas por adolescentes de 17 años de edad, $25 \%$ de 16 años de edad, mientras que el $19 \%$ de 15 años de edad. El $36 \%$ de infractores consumen alcohol, mientras que el $33 \%$ utiliza drogas; y el $31 \%$ consume ambas sustancias. Por lo que se puede concluir que los adolescentes infractores presentan adicciones hacia el alcohol y las drogas. El 53\% de infractores penales tienen el nivel educativo de secundaria, el $25 \%$ nivel primario, el $22 \%$ no tiene estudios. Los adolescentes infractores en su mayoría presentan estudios secundarios y un considerable número nivel primario. El $44 \%$ de infractores penales viven con su madre, el $19 \%$ vive con su padre, el $17 \%$ viven con ambos padres, el $14 \%$ viven con el tío y el $6 \%$ viven con abuelo. El $83 \%$ de infractores penales están en una situación económica precaria; mientras que el $17 \%$ señalan lo contrario.

Al $14 \%$ de infractores penales se les ha dictado una medida socio educativa de internación; al $33 \%$ libertad restringida; al 25\% libertad asistida; al $11 \%$ prestación de servicios a la comunidad; el $6 \%$ han sido absueltos y por último al $11 \%$ medida de amonestación. (Tabla 03).

Tabla 03. Medida socio educativa

\begin{tabular}{lrr}
\hline Medida socio educativa & $\mathrm{n}$ & $\%$ \\
\hline Prest. serv. a la comunidad & 4 & $11 \%$ \\
\hline Libertad restringida & 12 & $33 \%$ \\
\hline Libertad asistida & 9 & $25 \%$ \\
\hline Absuelto & 2 & $6 \%$ \\
\hline Amonestación & 4 & $11 \%$ \\
\hline Internación & 5 & $14 \%$ \\
\hline TOTAL & 36 & $100 \%$ \\
\hline
\end{tabular}

Respecto al cuestionario aplicado a los abogados, el $88 \%$ de los encuestados señalan que el entorno familiar se encuentra asociado a los adolescentes infractores de la ley penal en el distrito judicial de Tacna; y, el 12\% manifiesta lo contrario. El $83 \%$ afirma que también lo es el nivel educativo, el $79 \%$ las adicciones, el $76 \%$ la situación económica. El 83\% de los encuestados señalan que la mayoría de los adolescentes infractores cometen delitos contra el patrimonio y el $43 \%$ señalan que la mayoría cometen delitos en la modalidad de robo.

Asimismo, el $83 \%$ de los encuestados señalaron que los criterios para la determinación de las medida socioeducativas impuestas no son las más adecuadas para sancionar al adolescente infractor ya que el $89 \%$ señalan que las medidas socioeducativas de amonestación, prestación de servicios a la comunidad, libertad asistida, libertad restringida e internamiento no sirven para concientizar al menor en su convivencia social así como tampoco lo son las medidas de protección referidas al cuidado del hogar, incorporación de una familia sustituta, atención Integral a un establecimiento de protección especial.
El $78 \%$ de los encuestados señalan que las medidas socioeducativas de internamiento aplicadas son las más adecuadas para sancionar al adolescente infractor pero el $83 \%$ señalan que no resulta adecuado el tratamiento sentencial y jurisprudencial que se viene aplicando a los adolescentes infractores en la zona judicial de Tacna

En lo referente a la entrevista a los magistrados, se resume que el entorno familiar es el principal factor asociado a la infracción de la ley penal por los adolescentes infractores, ya que la mayoría de los adolescentes infractores provienen de familias disfuncionales, ya que viven con un solo progenitor, el cual no tiene pleno control del menor. El hecho de que las penas o medidas socioeducativas no son tan severas, hace que los menores infractores sean utilizados por personas mayores para cometer actos delictivos. Asimismo asumen que el principal factor económico que se asocia a la infracción a la ley penal por los adolescentes infractores es la situación de pobreza en la que viven los adolescentes infractores. Concluyen que los criterios para la determinación de la medida socioeducativas impuestos no son los más adecuados porque no se considera una 
adecuada evaluación del ambiente en que se desarrollan los menores infractores. Respecto a las medidas socioeducativas no privativas de libertad, los magistrados refieren no son las más adecuadas por cuanto el menor debe tener conciencia del hecho que ha cometido y para lo cual necesariamente tiene que reparar el daño, y esto se va a lograr con la justicia restaurativa, como se viene haciendo en otros distritos judiciales. Sugieren deberían implantarse como medida socioeducativas innovativas las escuelas militarizadas donde estén bien y salgan sólo los fines de semana y de las que existen las privativas de libertad como intervención

\section{DISCUSIÓN}

Durante los últimos años hemos sido testigos de actos de violencia juvenil en diversos lugares del Perú, y en la ciudad de Tacna y es por ello que con este trabajo de investigación de alguna manera sirva para erradicar este problema. Los principales factores que se encuentran asociados a la infracción de la ley penal cometida por los adolescentes, 2013-2016, son: sociales, económicos y jurídicos. Luego de analizar los datos del factor social y la infracción de la ley penal sobre los adolescentes, se analizó el entorno familiar, el $44 \%$ de infractores penales viven con su madre, otro $19 \%$ vive con su padre, el $17 \%$ viven con ambos padres, el $14 \%$ viven con el tío y el $6 \%$ viven con abuelo. Asimismo, es necesario señalar que estos resultados se ven contrastados con los resultados del cuestionario aplicado a los abogados y la entrevista aplicada a los magistrados. Cabe destacar que la infracción penal mayormente cometida es en los delitos contra el patrimonio en su modalidad de robo, utilizando para ello arma blanca, produciendo algunas veces lesiones en la víctima. En cuanto a las adicciones y la infracción de la ley penal se observa que el $19 \%$ de infractores consumen alcohol, mientras que el $33 \%$ utiliza drogas; y el $31 \%$ consume ambas sustancias. Los resultados tienen relación con lo manifestado por los abogados donde el $79 \%$ señalan que las adicciones se encuentran asociadas a los adolescentes infractores de la ley penal en el distrito judicial de Tacna.

Como dice Jhon Watson: "Ios niños son moldeados por sus ambientes, los padres tienen la responsabilidad de lo que llegarán a ser sus hijos", advirtiendo que los padres deben enseñar a sus hijos los buenos hábitos, lo que no ha sucedido en los casos investigados. Asimismo, las conductas antisociales, tanto del padre con sus familias, como la madre por las dificultades para mantener a los hijos o por la falta de atención, el hogar que no es atractivo para el adolescente ocasionando que no se sienta a gusto en él, como lo indica Hikal Carreon.
En cuanto al entorno educativo, como resultado de la investigación, los adolescentes tienen como nivel educativo, la secundaria. Comparto la opinión de Hikal Carreon cuando menciona que la educación escolar y familiar es considerada como uno de los medios más efectivos para prevenir lo antisocial y que el aprendizaje conductual para el estudio y el mejoramiento de la personalidad es una de las teorías de la personalidad más optimista. $\mathrm{L}$ o $\mathrm{s} d$ a t o $\mathrm{s}$ obtenidos permiten determinar que el entorno familiar tiene algún grado de afectación en los casos investigados; como también la relación de los padres con los profesores o tutores, la intervención de ellos en la escuela de padres u otras reuniones que se lleven a cabo en las instituciones educativas, la preocupación en el rendimiento escolar y las conductas de sus hijos como lo señala Trahtemberg Siederer. El resultado de la investigación se encuentra plasmada en las teorías criminológicas, en la cual la teoría de Anomia nos da ciertos alcances de forma relativa el comportamiento del adolescente infractor en la sociedad, esta teoría se inclina a los desbarajustes sociales que alteran la armonía social dejando a muchos adolescentes sin metas que alcanzar, que se sientan perdidos, desorientados ya que por un lado observan que personas de clases sociales altas tienen mejores condiciones de aquellos de clase social baja. Durkheim, señala que la anomia es un fenómeno social que empuja a los individuos a la desintegración y al no conformismo concluyendo con el delito.

Los profesionales y/o interesados en la problemática de los adolescentes infractores a la ley penal deben realizar estudios en relación a los hogares disfuncionales y con antecedentes de violencia familiar en el cual no habría un control debido y/o ausencia de control y supervisión de los padres con sus hijos. Los maestros de las distintas instituciones educativas deben analizar y reflexionar acerca de lo que debe incluirse en la formación del 
adolescente, para una debida conducta como alumno frente a sus compañeros de estudio. Considero que los comportamientos en la escuela entre los alumnos ha influido mucho, presentándose una crisis de autoridad, además no se presta una debida atención a las conductas de los alumnos, considerándola como algo propio de la edad, de la vida escolar, o que de alguna manera les sirva para defenderse o madurar, tenemos que dejar de pensar en ello y de esta forma evitar los malos tratos de algunos alumnos sobre otros más débiles ya que esto les daña tanto en su desarrollo social como en su personalidad. Por otro lado los medios de comunicación deben cumplir con el objetivo de que sus programas tengan un carácter educativo, cultural e informativo; desarrollar la idea de una cultura de no violencia, desarrollar una cultura de respeto a los derechos humanos y de las libertades fundamentales. Los operadores de justicia deben realizar un seguimiento a las medidas socioeducativas que imponen a los menores infractores y analizar si una educación técnica laboral sería buena para reinsertar a los adolescentes infractores en talleres y en convenios de microempresas. Ante el sistema judicial, es necesario primeramente una reflexión conjunta de todos los sectores involucrados, una coordinación de los poderes del Estado, de éstos entre sí y de éstos con la comunidad. El fracaso de todo acuerdo institucional depende de la integridad personal, si no se tienen ideales de justicia, no estamos preparados para el ejercicio de estas responsabilidades. Asimismo, las motivaciones del infractor penal son múltiples y cada caso es único, se deben establecer modelos integrales que consideren estos múltiples factores para encontrar la solución acorde a la realidad.

Presentado: Marzo 2017

Aceptado: Junio 2017

\section{REFERENCIAS BIBLIOGRÁFICAS}

Arias, J. (1952). Derecho de Familia (Segunda ed.). Buenos Aires: Kraft.

Defensoria Del Pueblo. (Julio de 2012). www.incipp.org.pe. (1. Edición, Editor, \& I. D. 157-2012/DP, Productor) Obtenido de Defensoría del Pueblo

Diaz de Guijarro, E. (1943). La Familia, la Sociedad Civil y el Estado en el Sistema de Hegel. Buenos Aires: Antología Jurídica.

Hernandez, C. (2005). El debido proceso y la justicia penal juvenil. Tesis, Universidad Nacional Mayor de San Marcos, Perú - Lima.

Hikal, W. S. (2005). Criminología psicoanalítica, conductual y del desarrollo (Primera ed.). Mexico: ISBN.

Hinostroza, A. (1999). Derecho de Familia (Tercera ed.). (A. J. Paredes Galvan, Ed.) Perú: San Marcos.

Kemelmajer, A. (2004). Justicia Restaurativa. Argentina: Rubinzal - Culzoni Editores.
Miranda, P. (2011). Realidades y desafios en la intervención del trabajador social con adolescentes infractores de la ley penal. Tesis, Universidad Academia de Humanismo Cristiano, Chile-Santiago.

Reyna, A. (2011). Aplicación de la justicia restaurativa en adolescentes infractores en el Canton Ibarra en el año 2011. Tesis, Escuela de Jurisprudencia de la Pontificia Universidad Católica del Ecuador, Ecuador - Ibarra.

Trahtemberg, L. (1999). Educación para el Tercer Milenio. Lima, Perú: Asociación EditorialBruño.

Wiese, R. (2008). Jóvenes que cometen delitos severos. Tesis, Universidad Nacional de La Plat a, Buenos A ires - L a Plata.www.adolescenciayjuventud.org. (febrero de 2013) 